\title{
Asymmetry in the triplet 3p-4s Mg lines in cool DZ white dwarfs
}

\author{
N. F. Allard ${ }^{1,2}$, T. Leininger ${ }^{3}$, F. X. Gadéa ${ }^{3}$, V. Brousseau-Couture ${ }^{4}$, and P. Dufour ${ }^{4,5}$ \\ ${ }^{1}$ GEPI, Observatoire de Paris, PSL Research University, UMR 8111, CNRS, Université Paris Diderot, Sorbonne Paris Cité, \\ 61 avenue de l'Observatoire, 75014 Paris, France \\ e-mail: nicole.allard@obspm.fr \\ 2 Institut d'Astrophysique de Paris, UMR 7095, CNRS, Université Paris VI, 98bis boulevard Arago, 75014 Paris, France \\ ${ }^{3}$ Laboratoire de Physique et Chimie Quantique, Université de Toulouse (UPS), UMR 5626, and CNRS, 118 route de Narbonne, \\ 31400 Toulouse, France \\ ${ }^{4}$ Département de Physique, Université de Montréal, Montréal, Québec H3C 3J7, Canada \\ 5 Institut de Recherche sur les Exoplanètes (iREx), Université de Montréal, Montréal, Québec H3C 3J7, Canada
}

Received 24 November 2015 / Accepted 25 January 2016

\begin{abstract}
The purpose of the present work is to make an exhaustive study of the line shape of the triplet $3 \mathrm{p}-4 \mathrm{~s} \mathrm{Mg} \mathrm{line} \mathrm{(Mgb} \mathrm{triplet),} \mathrm{which} \mathrm{is}$ perturbed by $\mathrm{He}$ in the extreme physical conditions found in the cool atmosphere of DZ white dwarfs. This study is undertaken by inferring both a unified theory of spectral line broadening and ab initio potential energies. Cool white dwarfs require a specific treatment for line broadening owing to the high helium densities that are involved. Beyond the conventional symmetrical Lorentzian core at low density, we show that the line profiles are asymmetrical and have significant additional contributions on the short wavelength side. This blue asymmetry is a consequence of low maxima in the corresponding Mg-He potential energy difference curves at short and intermediate internuclear distances. The new profiles are shown to provide a good fit to an SDSS (Sloan Digital Sky Survey) observation.
\end{abstract}

Key words. line: profiles - stars: atmospheres - white dwarfs

\section{Introduction}

Heavy elements are not expected to be found at the surface of old white dwarfs owing to their very high surface gravity. Indeed, metals settle on timescales that are much shorter than the cooling age of these stars, thus leaving behind a pure hydrogen/helium photosphere (Paquette et al. 1986). Yet, traces of heavy elements, such as calcium, magnesium, and iron are commonly detected at the surface of many old white dwarfs (see Koester et al. 2014, and references therein), indicating that an external source must have recently replenished the photosphere. It is now accepted that this source is the accretion from a disk that was formed by the tidal disruption of one or many rocky objects that passed too close to the white dwarf, most likely asteroids or minor planets (Jura \& Young 2014; Koester et al. 2014). Hence, these "polluted" white dwarfs can be used as a probe to characterize the chemical composition of rocky material that once orbited mainsequence stars.

To fully exploit the potential of these objects as planetary probes, we need to first obtain accurate photospheric abundances. Indeed, because of the relatively low opacity of the helium atmospheres at lower effective temperatures, photons that escape the star originate from deeper layers where the density is very high. Under these conditions, the emiting atoms can experience multiple simultaneous perturbations with the surrounding helium atoms. A reliable calculation of the line profiles that is applicable in all parts of the line at all densities is the Anderson semi-classical theory (Anderson 1952), which utilizes the Fourier transform (FT) of an autocorrelation function.

The metals most commonly identified at white dwarf's photospheres are calcium, magnesium, and iron. Calculating their abundances requires accurate description of the line profiles. However, there is a reliance on simplified models of linebroadening physics that remains in most of the code that is in current use. These models, typically use either Lorentzian or quasistatic van der Waals profiles with adjusted empirical parameters.

This paper is a continuation of our studies of sodium and ionized calcium resonance lines that are perturbed by helium (Allard \& Alekseev 2014; Allard 2013; Allard et al. 2014). We consider the satellite band that is associated with the transition between the $3 \mathrm{p}$ and the $4 \mathrm{~s}$ levels of a magnesium atom, which is perturbed by dense helium plasma. The purpose of this investigation is to establish the link between the strong asymmetric spectral features that are observed in cool DZ white dwarfs and the existence of a very close blue satellite. The asymmetrical shapes of spectral lines have been extensively investigated for many years because of their importance in experimental and theoretical work (Allard \& Kielkopf 1982). The asymmetry in the $\mathrm{Mg}$ profile was incorrectly identified as being due to quasistatic broadening by Wehrse \& Liebert (1980).

The triplet 3p-4s line profiles of Mg perturbed by helium, are calculated for the physical conditions encountered in the atmospheres of cool white dwarfs. Starting with the Anderson (1952) theory, suitably generalized to include degeneracy, a unified theory of spectral line broadening (Allard et al. 1999) has been developed to calculate neutral atom spectra, given the interaction and the radiative transition moments of relevant states of the radiating atom with other atoms in its environment. Line profile calculations in our work have been done using accurate ab initio potential energies that take into account the long range part (Sect. 2). The theory (Sect. 3) and interpretation (Sect. 4) are 
similar to that previously reported in (Allard et al. 2009, 2011, 2012; Allard 2012) for interpreting observations of a corona discharge in liquid He. Several theoretical computed profiles are used to illustrate the evolution of the line and satellite with temperatures (Sect. 5). Shift, broadening, and asymmetry are discussed in terms of the evolution of the spectral line shapes with density in Sect. 6. Finally an astrophysical application for a cool DZ white dwarf is reported in Sect. 7.

\section{MgHe diatomic potentials}

Significant progress in the description of $\mathrm{Mg}-\mathrm{He}$ singlet and triplet potential energies has been achieved by Alekseev $2014^{1}$, but in a small range, $R \sim 2.5$ to 20 a.u., where $R$ denotes the internuclear distance between the radiator and the perturber. To predict the impact core shift and width, the long range part of the potential energies needs to be accurately determined.

The ab initio calculations of the potentials were made using a large core pseudopotential (Fuentealba et al. 1983) for Mg complemented by operatorial core polarisation potential (CPP), with the MOLPRO package (Werner et al. 2012). For Mg, we built a large basis set that was inspired by the standard (pseudopotential) basis set (Fuentealba et al. 1982) and the one used in rather high Rydberg state calculations (Khemiri et al. 2013) leading to a 10s9p6d3f3g basis set. For He, the huge 30s17p10d6f3g basis set of Deguilhem and coworkers was used (Deguilhem et al. 2009). State-specific orbitals were obtained from CASSCF calculations (Werner \& Knowles 1985), where the active space consisted of four electrons distributed in all orbitals up to the $4 \mathrm{~s}$ of $\mathrm{Mg}$ (i.e., a CAS 4,6). These orbitals were then used in subsequent MRCI (Werner \& Knowles 1988) calculations to obtain the potential energy curves, as well as static and transition dipole moments for all permitted transitions. The potential curves have been computed using a dense grid of internuclear points ranging from $R=4$ to 500 a.u. Potential energy curves $V(R)$ for the $\mathrm{MgHe}$ molecule involved in the transition between the $3 \mathrm{p}$ and the $4 \mathrm{~s}$ levels are shown in Fig. 1.

Spin-orbit interaction is taken into account using the Cohen Schneider scheme where the atomic SO coupling is generalised to the molecule (Cohen \& Schneider 1974). It has been shown to work very well for heavy rare gases ionic clusters such as $\mathrm{Kr}_{n}^{+}$ and $\mathrm{Xe}_{n}^{+}$(Hrivnak et al. 2005). Here, for a $n \mathrm{~s} n^{\prime} \mathrm{p}$ scheme, two matrices need to be diagonalised, respectively for $\Omega=0$ and $\Omega=1$, while for $\Omega=2$, there is just an energy shift of the ${ }^{3} P_{\Pi}$ energy:

$\Omega=0$

$\left(\begin{array}{cccc}E\left({ }^{3} P_{\Pi}\right)-\alpha & \alpha & 0 & \alpha \\ \alpha & E\left({ }^{3} P_{\Sigma}\right) & \alpha & 0 \\ 0 & \alpha & E\left({ }^{3} P_{\Pi}\right)-\alpha & -\alpha \\ \alpha & 0 & -\alpha & E\left({ }^{1} P_{\Sigma}\right)\end{array}\right)$

$\Omega=1$

$\left(\begin{array}{ccc}E\left({ }^{3} P_{\Pi}\right) & \alpha & \alpha \\ \alpha & E\left({ }^{3} P_{\Sigma}\right) & -\alpha \\ \alpha & -\alpha & E\left({ }^{1} P_{\Pi}\right)\end{array}\right)$

$\Omega=2$

$E\left({ }^{3} P_{\Pi}\right)+\alpha$.

1 https://www.researchgate.net/profile/
Vadim-Alekseev/publications

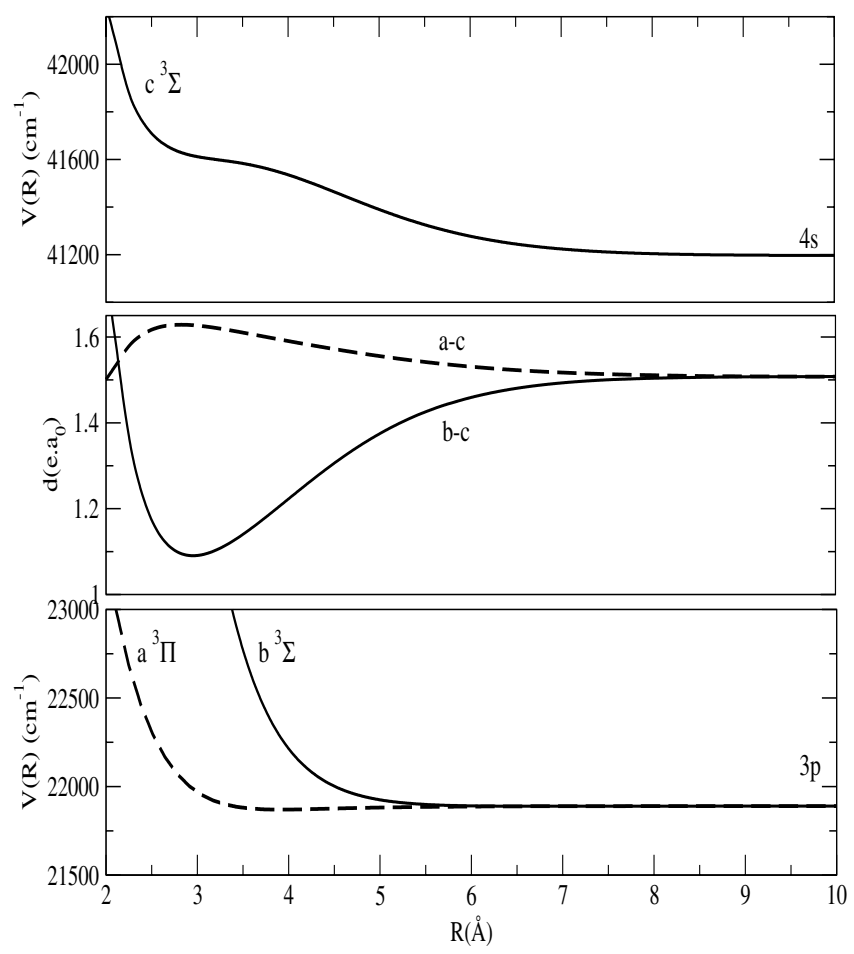

Fig. 1. Potential energies for the $a, b$, and $c$ states of the $\mathrm{Mg}$ He molecule without SO coupling. $4 \mathrm{~s} c^{3} \Sigma$ (full line), $3 \mathrm{p} b^{3} \Sigma$ (full line), $3 \mathrm{p} a^{3} \Pi$ (dashed line), and transition dipole moment $c-b$ (full line), $c-a$ (dashed line).

Here, $\Omega$ corresponds to the good quantum number in the Hund case $c(\Omega=\Lambda+\Sigma)$ for the molecule and corresponds to $M_{J}=$ $M_{L}+M_{S}$ for the atoms.

For $\alpha$ we have taken $20.2573 \mathrm{~cm}^{-1}$, to recover the correct $J=2-J=0$ atomic splitting. Asymptotically, the ${ }^{3} P_{\Sigma}$ and ${ }^{3} P_{\Pi}$ are degenerated and the matching between the atomic and molecular energies (degeneracies are recalled in paranthesis) is given in Table 1 . The degeneracy is lifted by the SO coupling and the states at $+\alpha,-\alpha$ and $-2 \alpha$ match the atomic states, $J=2$, $J=1$, and $J=0$, respectively.

For general $\mathrm{R}$, this degeneracy is lifted by molecular interactions. The molecular states are now: $a: 1^{3} \Pi, b: 1^{3} \Sigma, c: 2^{3} \Sigma$. The He atom remains closed shell, therefore the occupation in $\mathrm{Mg}$ mainly controls the identification of the states and the labelling that relates to the $\mathrm{Mg}$ atoms still makes sense. The resulting potentials are given in Fig. 2 and compared with the potentials without spin-orbit coupling. Spin-orbit coupling has a huge effect and leads to two very repulsive states correlating to $J=2$, two curves with double-well shapes correlating to $J=1$ and $J=0$, while the two last potentials remain very similar to the ${ }^{3} \Pi$ state.

On the other hand, the main difference at short distances between the repulsive walls of the $a$ and $b$ potentials remains after spin-orbit coupling. For the various $\Omega$, all potentials remain at short distances that are either close to the $a$ (the two lowests for $\Omega=0$, the lowest for $\Omega=1$ and the $\Omega=2$ ) or to the $b$ (the highest for $\Omega=0$ and $\Omega=1$ ) repulsive potentials. We will see the effect on the potential differences in the next section and, consequently, on the line profiles of the different components.

\section{Theoretical profiles}

Foreign-gas pressure-broadening of spectral lines is due to the difference in the interaction energies of the optical atom (in the 
Table 1. Molecular to atomic asymptotic energy matching.

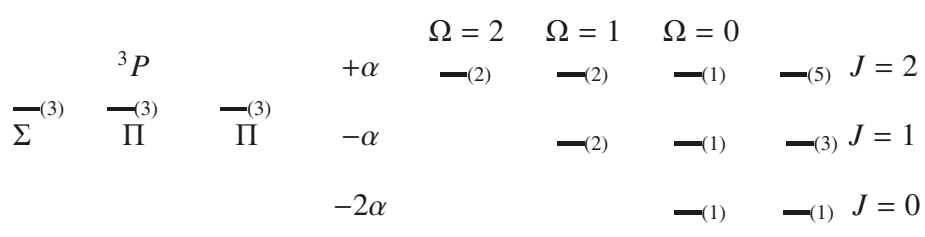

$$
\begin{aligned}
& \text { Molecular without SO } \quad \text { Molecular with SO Atomic }
\end{aligned}
$$

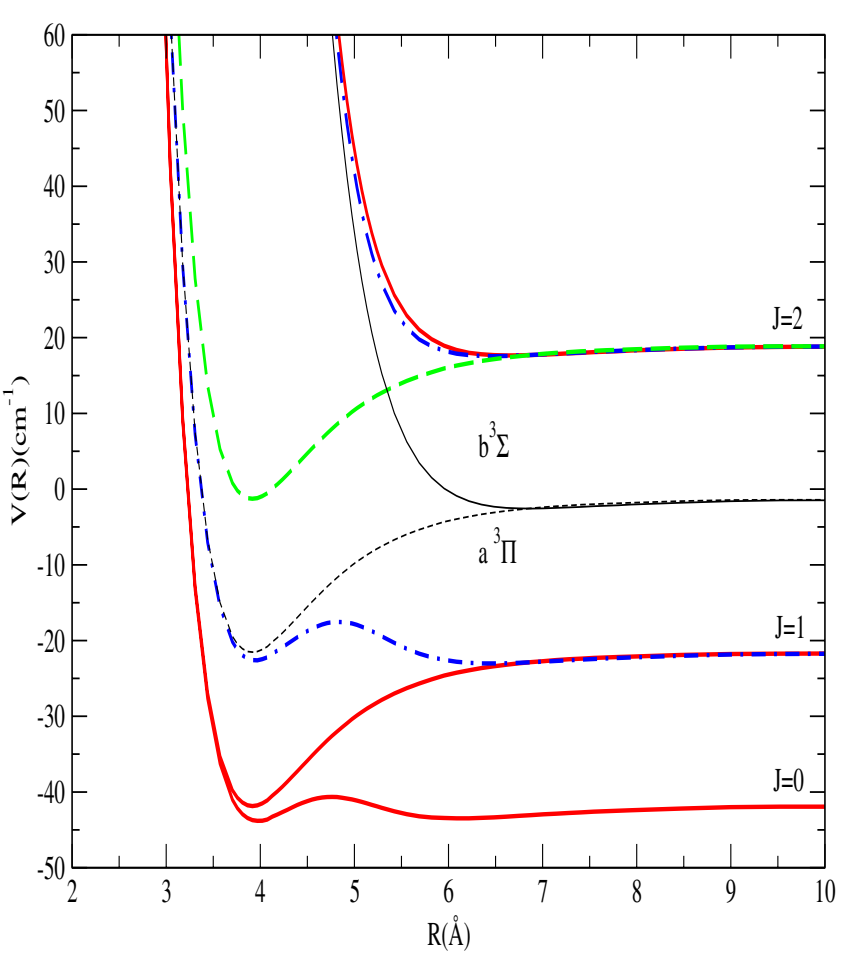

Fig. 2. Potential curves correlated with the $3 p$ states without (black) and with inclusion of spin-orbit coupling (red/full: $\Omega=0$, blue/dash-dot: $\Omega=1$ and green/dash: $\Omega=2$ ) of the $\mathrm{Mg}-\mathrm{He}$ molecule. The energies are given relative to the potentials without spin-orbit coupling. The fine structure separation is $20.2573 \mathrm{~cm}^{-1}$.

initial and final states involved in the radiating or absorbing process) and a foreign colliding atom over a wide range of distances.

As a first approach (Leininger et al. 2015) we used potential energies of the $3 p$ state without SO coupling (Fig. 1). At high He density, the lines $3 \mathrm{p}^{3} P_{2,1,0}$ are not resolved, the spin-orbit was not included in our calculations, we were typically in the Hund case $b$. The line profile depends only on the two individual transitions $a 3 \mathrm{p}^{3} \Pi \rightarrow c 4 \mathrm{~s}^{3} \Sigma$ and $b 3 \mathrm{p}^{3} \Sigma \rightarrow c 4 \mathrm{~s}^{3} \Sigma$.

The prediction of the shape of the $3 \mathrm{p} \rightarrow 4 \mathrm{~s}$ line required us to study $\Delta V(R)$, as shown in Fig. 3. The difference potential maxima are respectively 400 and $165 \mathrm{~cm}^{-1}$ for $a-c$ and $b-c$ transitions.

The unified profiles are the Fourier transforms of the autocorrelation functions as given by Eq. (121) of Allard et al. (1999) in which the contributions from the two components of the transition enter with their statistical weights. The unified theory predicts that there will be line satellites centered periodically at frequencies corresponding to integer multiples of the extrema of $\Delta V$.

Figure 4 shows the individual components for comparison, weighted as if they were the only contribution to the profile.

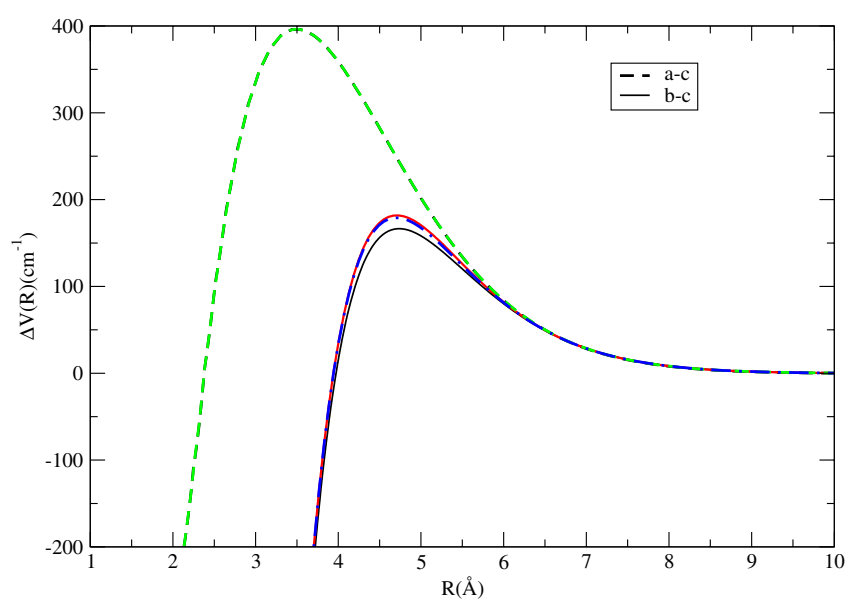

Fig. 3. $\Delta V$ for the transitions contributing to $3 p \rightarrow 4$ s line without $\mathrm{SO}$ (black lines): $3 \mathrm{p}^{3} \Sigma \rightarrow 4 \mathrm{~s}^{3} \Sigma$ and $3 \mathrm{p}^{3} \Pi \rightarrow 4 \mathrm{~s}^{3} \Sigma$. All the other $\Delta V$ for the components $J=2$ are superimposed (red/full: $\Omega=0$, blue/dashdot: $\Omega=1$ and green/dash: $\Omega=2$ ).

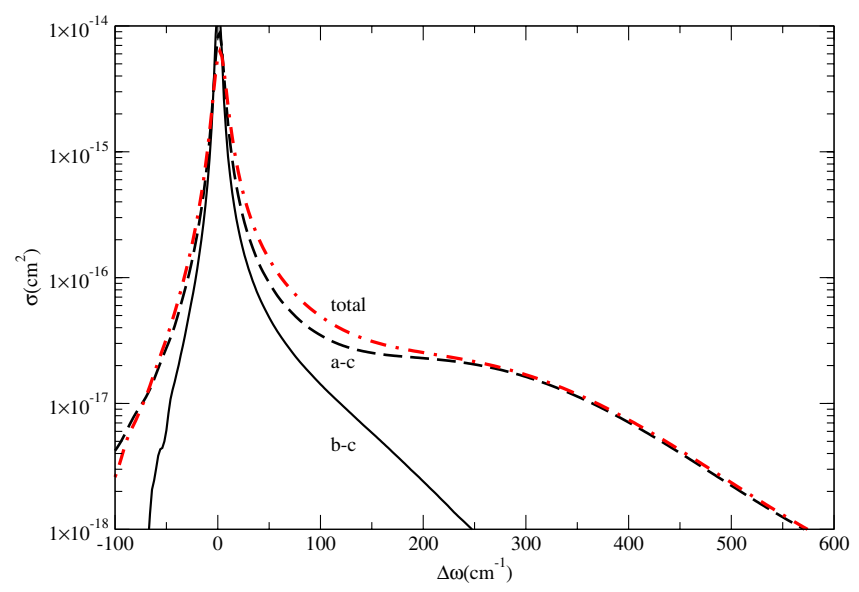

Fig. 4. Individual components of the line profile, neglecting the SO coupling, compared to the total profile at $T=6000 \mathrm{~K}$ and for $n_{\mathrm{He}}=$ $10^{20} \mathrm{~cm}^{-3}$

The distinct wide shoulder at about $240 \mathrm{~cm}^{-1}$ owing to the $3 \mathrm{p}^{3} \Pi \rightarrow 4 \mathrm{~s}^{3} \Sigma$ transition, yields a line satellite in the blue wing that is about $5120 \AA$ (Fig. 3 of Leininger et al. 2015). The other maximum at $\Delta V=165 \mathrm{~cm}^{-1}$ does not give the slightest hint of a blue shoulder, the corresponding individual profile simply appears to have a blue asymmetry.

To provide opacity data ${ }^{2}$ at any He density, we will consider the different components $J_{n}$ of the triplet using the potentials with inclusion of spin-orbit coupling (Fig. 2). As mentioned before, all potentials remain at short distances either close to the

2 Complete tables are available on request from NFA. 


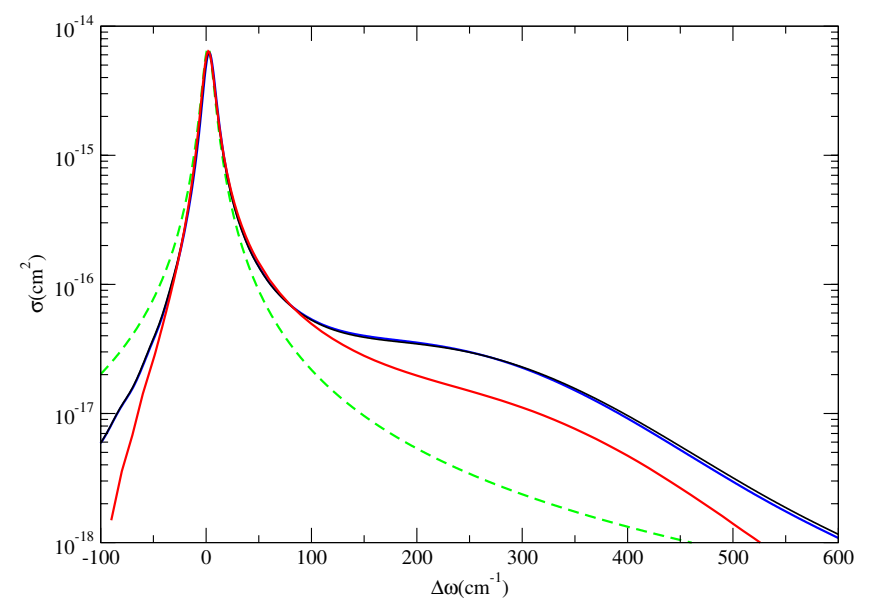

Fig. 5. Theoretical absorption cross sections of the components $J_{0}$ (blue line), $J_{1}$ (black line), and $J_{2}$ (red line) compared to the Lorentzian profile for $J_{2}$ (green/dash line). The density of perturbers is $n_{\mathrm{He}}=$ $10^{20} \mathrm{~cm}^{-3}$. The temperature is $6000 \mathrm{~K}$.

Table 2. Half-width at half maximum $(w)$ and shift $(d)\left(10^{-20} \mathrm{~cm}^{-1} /\right.$ $\mathrm{cm}^{-3}$ ) of the components of the triplet lines at $T=6000 \mathrm{~K}$.

\begin{tabular}{lccc}
\hline \hline Lorentzian parameters & $J_{0}$ & $J_{1}$ & $J_{2}$ \\
\hline$w$ & 5.37 & 5.37 & 5.57 \\
$d$ & +2.79 & +2.30 & +1.89 \\
\hline
\end{tabular}

$a$ and $b$ potentials, the values of $\Delta V$ for $J_{2}$ remain almost unchanged (Fig. 3). We did not plot $\Delta V$ for $J_{0}$ and $J_{1}$ since they are all close to $a-c$. Figure 5 shows the line shape of the different components $J_{n}$ of the triplet at $n_{\mathrm{He}}=10^{20} \mathrm{~cm}^{-3}$ and $T=6000 \mathrm{~K}$. The main change concerns the $J_{2}$ component. Because of the smaller relative weight of the contribution of the $a-c$ transition, the line satellite is not well-pronounced compared to those appearing in the near wings of $J_{0}$ and $J_{1}$ which are strictly due to $a-c$. Since we are considering a low pressure, the core of the line is described adequately by a Lorentzian, and because of the $\log$ scaling in Fig. 5, the Lorentzian is plotted only for $J_{2}$. In Table 2, we report our computed half-widths at half maximum (HWHM) and shift in the impact approximation for the different components $J_{n}$ of the triplet at $T=6000 \mathrm{~K}$.

\section{The influence of close blue satellite on spectral line shape}

Several helium-rich white dwarf stars show an asymmetry in the Mgb triplet (many examples can be found in Koester et al. 2011), indicating that the physical conditions present in these stars are such that the impact approximation is no longer valid. Figure 6 shows a typical temperature and pressure structure for one such star, SDSS 1535+1247, the brightest object in the Koester et al. (2011) sample. With densities well above $10^{20} \mathrm{~cm}^{-3}$ in the line forming region ( $\log \tau_{R} \sim-3$ to 0 ), the possibility of several atoms interacting strongly is high, and this plays a role in the wavelength of the line center, e.g. the shift of the line, as well as the continuum generated far from the line center. This is indeed what is observed in the spectra of SDSS 1535+1247 (see Fig. 13), where the asymmetric blue wing of the $\mathrm{Mg}$ triplet can easily be appreciated.

In the next section, we will evaluate collisional profiles of the different components $J_{n}$ of the triplet for relevant temperatures and densities that are appropriate for modeling helium-rich white
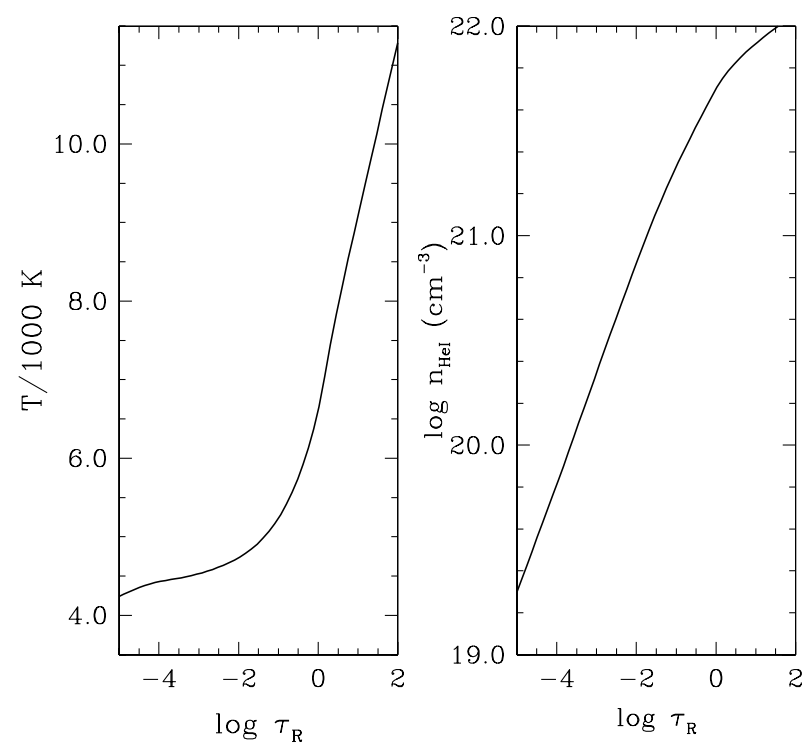

Fig. 6. Temperature and He density as a function of Rosseland mean optical depth assuming SDSS 1535+1247 atmospheric parameters (Koester et al. 2011).

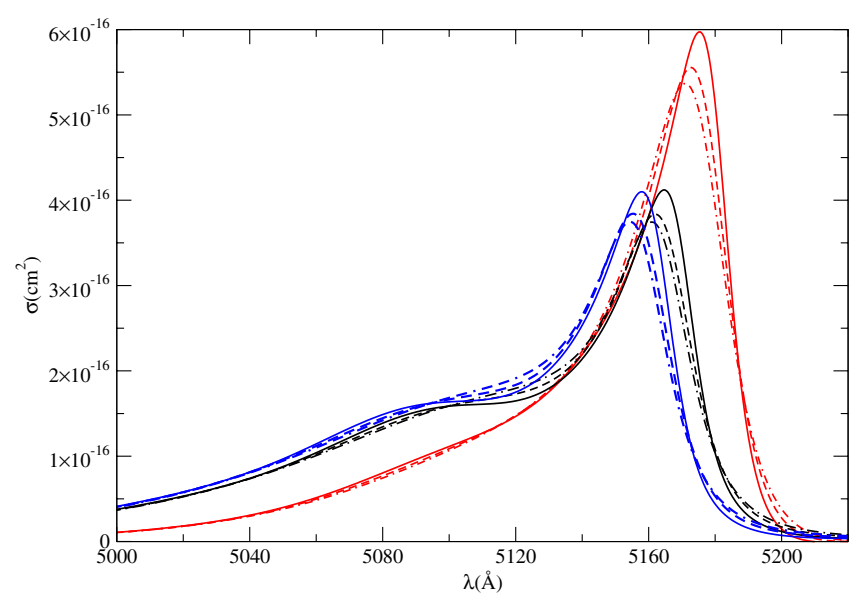

Fig. 7. Variation of the line profile with temperature for $n_{\mathrm{He}}=10^{21} \mathrm{~cm}^{-3}$ for the 3 components $J_{0}$ (blue line), $J_{1}$ (black line), and $J_{2}$ (red line), $4000 \mathrm{~K}$ (full line), $6000 \mathrm{~K}$ (dashed line), and $8000 \mathrm{~K}$ (dashed dotted line).

dwarf stars. For such high helium density the collisional effects should be treated by using the unified theory presented in Allard et al. (1999) to take into account simultaneous collisions with more than one perturbing atom. The traditional separation of line profiles into a core region to be treated by the impact approximation and a wing region to be treated by the quasistatic approximation fails to be satisfactory when very close line satellites lead to an asymmetrical shape of the line with increasing perturber density. This asymmetry in the Mgb triplet was uncorrectly identified as being due to quasistatic broadening by Wehrse \& Liebert (1980) and used in many astrophysical modelings (Kawka et al. 2004; Koester et al. 2011).

\section{Temperature dependence of the line satellite}

Collisional line profiles were evaluated for $n_{\mathrm{He}}=1 \times 10^{21} \mathrm{~cm}^{-3}$ from 4000 to $8000 \mathrm{~K}$, the line wings shown in (Fig. 7) are almost unchanged with increasing temperature. While the position 
N. F. Allard et al.: Asymmetry in the triplet 3p-4s Mg lines in cool DZ white dwarfs

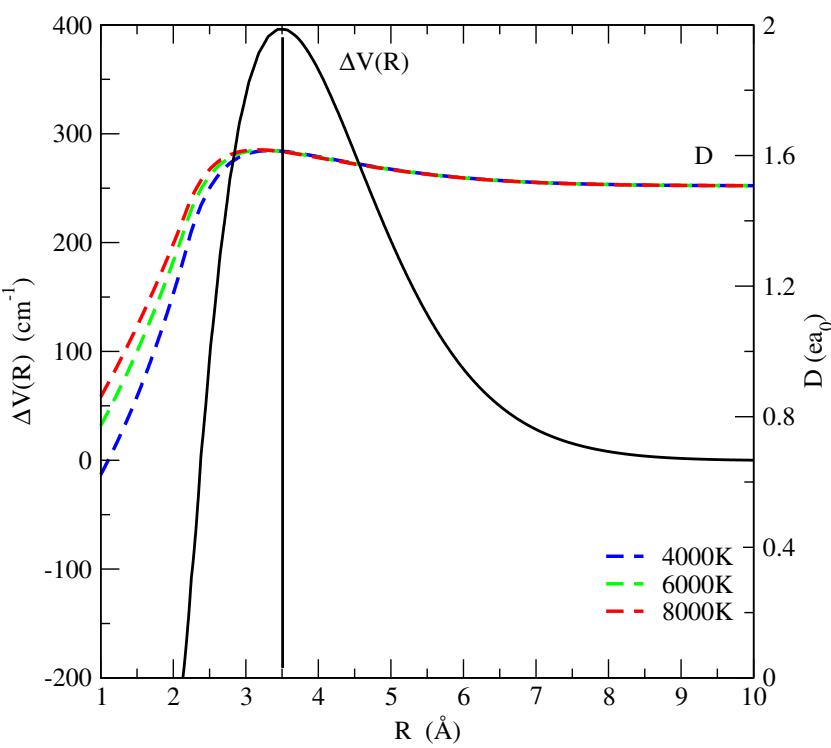

Fig. 8. Difference potential energy $\Delta V(R)$ in $\mathrm{cm}^{-1}$ (black line) and the temperature dependence of modulated dipole $D(R)$ (color lines) corresponding to the $3 \mathrm{p} a \rightarrow 4 \mathrm{~s} c$ transition.

of the line satellites critically depends on the interaction potential, their strength depends on both the interaction potentials and the radiative dipole moments, $D\left(R_{\text {ext }}\right)$, in the internuclear region where the line satellite is formed. In Allard et al. (1999) we defined $\tilde{d}_{e e^{\prime}}(R(t))$ as a modulated dipole

$D(R) \equiv \tilde{d}_{e e^{\prime}}[R(t)]=d_{e e^{\prime}}[R(t)] e^{-\frac{\beta}{2} V_{e}[R(t)]}$,

where $\beta$ is the inverse temperature $(1 / k T)$. Here $V_{e}$ is either the $a$ or $b$ states since we consider absorption profiles due to $a-c$ and $b-c$ transitions. An examination of Fig. 8 shows that the $5080 \AA$ line satellite is formed around $R_{\text {ext }}=3.5 \AA$. The flat dependence in $D(R)$ throughout this region is shown to not alter the amplitude of the satellite (Fig. 7). In this range of temperatures, the strength of the blue wing is not sensitive to the temperature.

\section{Density dependence of the collisional profiles}

In Figs. 9-11 we show the variation of the absorption cross section at $6000 \mathrm{~K}$ with increasing helium density. There is a dramatic change when the density gets larger than $10^{21} \mathrm{~cm}^{-3}$.

\subsection{Study of the line parameters}

The simplest way of characterizing a line shape is in terms of the parameters, $w$, the full width at half maximum (FWHM), $d$, the shift, and the asymmetry (which is computed by taking the ratio of the width at half of the maximum intensity of the blue side to that of the red side). The impact approximation is widely used for describing the central region of pressure-broadened spectral lines at low densities.

We have studied the variations of the line parameters versus the density for $T=6000 \mathrm{~K}$ for the $J_{0}$ and $J_{2}$ components. To determine these parameters, several theoretical unified profiles have been computed to illustrate the complicated evolution of the triplet line with gas pressure. Figure 12 allows us to understand the complex variation of the line profiles and the line parameters. At low density the core of the theoretical profile is not affected by the blue satellite, hence the width changes linearly with density,

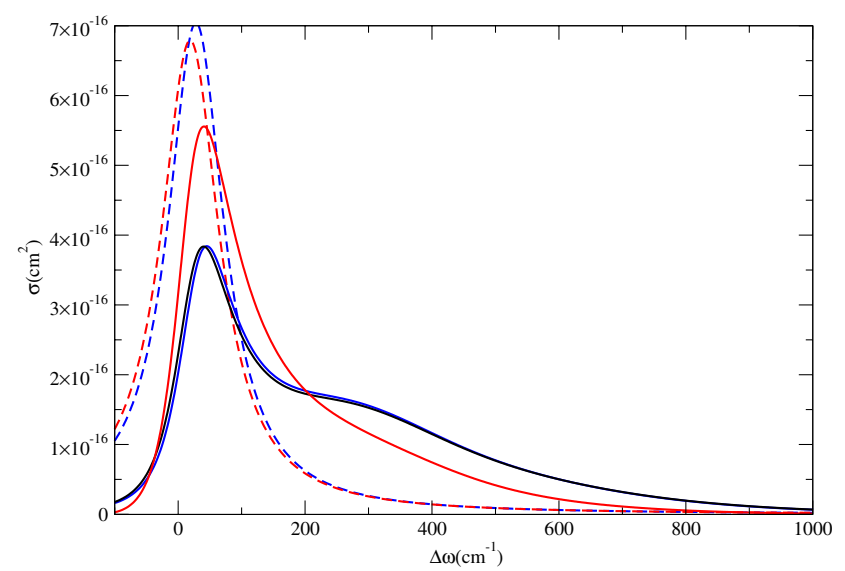

Fig. 9. Theoretical absorption cross sections of the components $J_{0}$ (blue line), $J_{1}$ (black line), and $J_{2}$ (red line) compared to the Lorentzian profiles for $J_{0}$ (blue/dash line) and for $J_{2}$ (red/dash line). The density of perturbers is $n_{\mathrm{He}}=10^{21} \mathrm{~cm}^{-3}$. The temperature is $6000 \mathrm{~K}$.

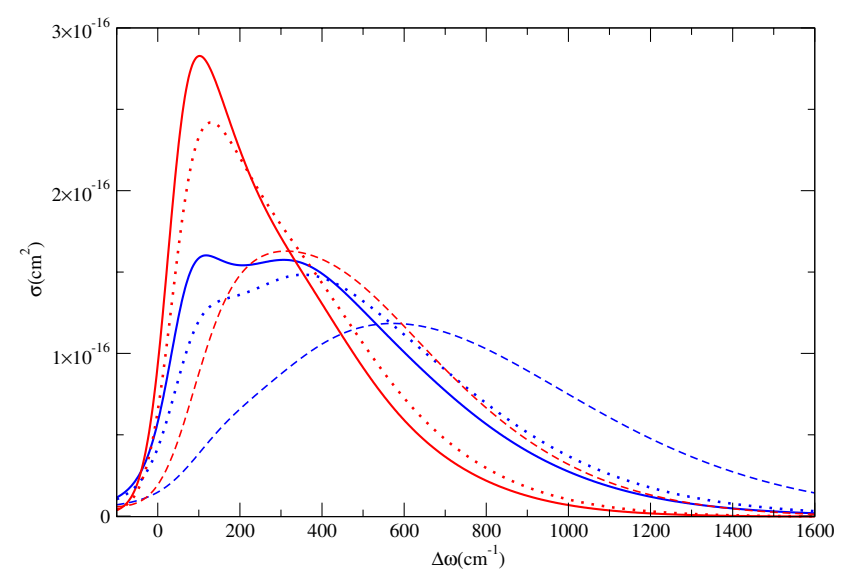

Fig. 10. Evolution of the unified profiles of the components $J_{0}$ (blue lines) and $J_{2}$ (red lines) with increasing helium density for $n_{\mathrm{He}}=1.75 \times$ $10^{21}$ (full line), $2 \times 10^{21}$ (dotted line) and to $3 \times 10^{21} \mathrm{~cm}^{-3}$ (dashed line), $T=6000 \mathrm{~K}$.

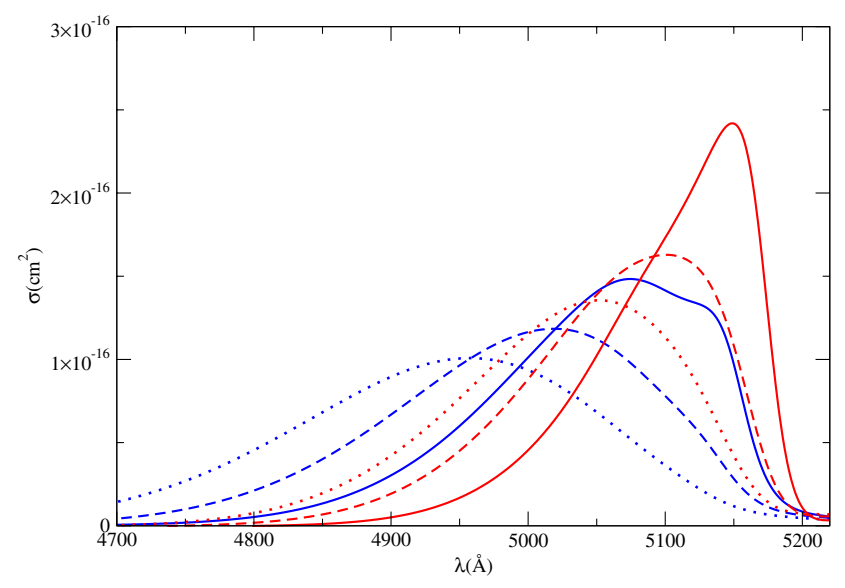

Fig. 11. Evolution of the unified profiles of the components $J_{0}$ (blue lines) and $J_{2}$ (red lines) with increasing helium density for $n_{\mathrm{He}}=2 \times 10^{21}$ (full line), $3 \times 10^{21} \mathrm{~cm}^{-3}$ (dashed line) and $4 \times 10^{21} \mathrm{~cm}^{-3}$ (dotted line), $T=6000 \mathrm{~K}$.

the same is true for the shift and, the asymmetry is close to one, like a Lorentzian. We show that striking general features of the shift, width and asymmetry curves occur when the pressure is high enough to blend the satellite and the line core (Figs. 9-11). 


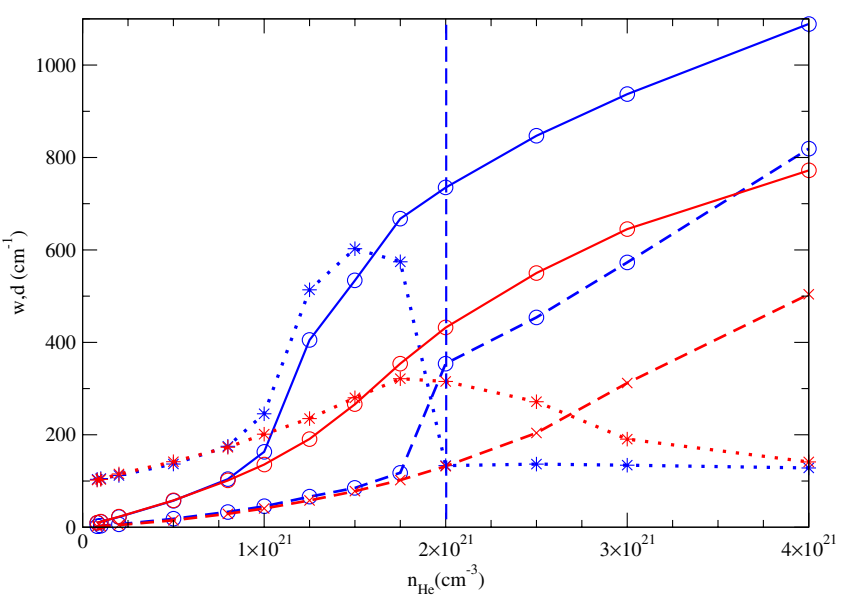

Fig. 12. Variation of the width (full line), shift (dashed line) and asymmetry $\times 100$ (dotted line) in the He density range $n_{\mathrm{He}}=5 \times 10^{19}$ to $4 \times 10^{21} \mathrm{~cm}^{-3}$ for $T=6000 \mathrm{~K} . J_{0}$ (blue lines) and $J_{2}$ (red lines).

\subsection{Study of the $J_{0}$ component}

The line satellite becomes higher than the main line for $\mathrm{n}_{\mathrm{He}}=$ $2 \times 10^{21} \mathrm{~cm}^{-3}$. Hence, a "discontinuity" appears on the shift; the shift is now being measured from the satellite peak rather than the line peak. An analogous "discontinuity" appears on the asymmetry curve. At this point, the width is measured relative to the half-magnitude of the satellite. The situation is now reversed. The satellite is now regarded as the main line and is broadened by a decreasing "satellite", when $n_{\mathrm{He}}=4 \times 10^{21} \mathrm{~cm}^{-3}$ the main line has totally disappeared (Fig. 11).

The reason for this transition to very non-linear behavior is that we are first measuring the width proper at lower density. Then, as the density increases and the satellite grows to more than one half the height of the line, we are measuring the combined width of line and satellite.

Moreover, we have known for a long time, since the pioneering work of Cartan \& Hindmarsh (1969), that multiple satellites can be observed experimentally at very high densities. A definite observation of multiple perturber satellites was reported in Kielkopf \& Allard (1979); they lead to a complicated evolution of the line parameters. In previous papers (Allard 1978; Allard \& Biraud 1980; Gilbert et al. 1980; Royer 1980), a theoretical model based upon a simple square-well potential was developed. This model represented the first step to calculations involving more realistic potentials. Even though simple, the model at that stage of development nevertheless provided one with a means of clearly understanding many features in the experimental parameter curves (Ch'en \& Garrett 1966; Ch'en et al. 1967, 1969; Garrett \& Ch'en 1966; Garrett et al. 1967; Tan \& Ch'en 1970).

\subsection{Study of the $J_{2}$ component}

Figures 9-11 reveal that the development of the blue wing leads to the line being overwhelmed by the line satellite, when the density is about $1.75 \times 10^{21} \mathrm{~cm}^{-3}$. When the density reaches $2 \times$ $10^{21} \mathrm{~cm}^{-3}$, the satellite completely blends with the line seen in Fig. 10. This is a smooth transition for that component compared to the discontinuities observed for $J_{0}$. As emphasized in Sect. 3, the small relative weight of the $3 \mathrm{p} a \rightarrow 4 \mathrm{~s} c$ transition gives an apparently featureless blue wing. Nevertheless the development of the close satellite leads to the change in the slope of the width

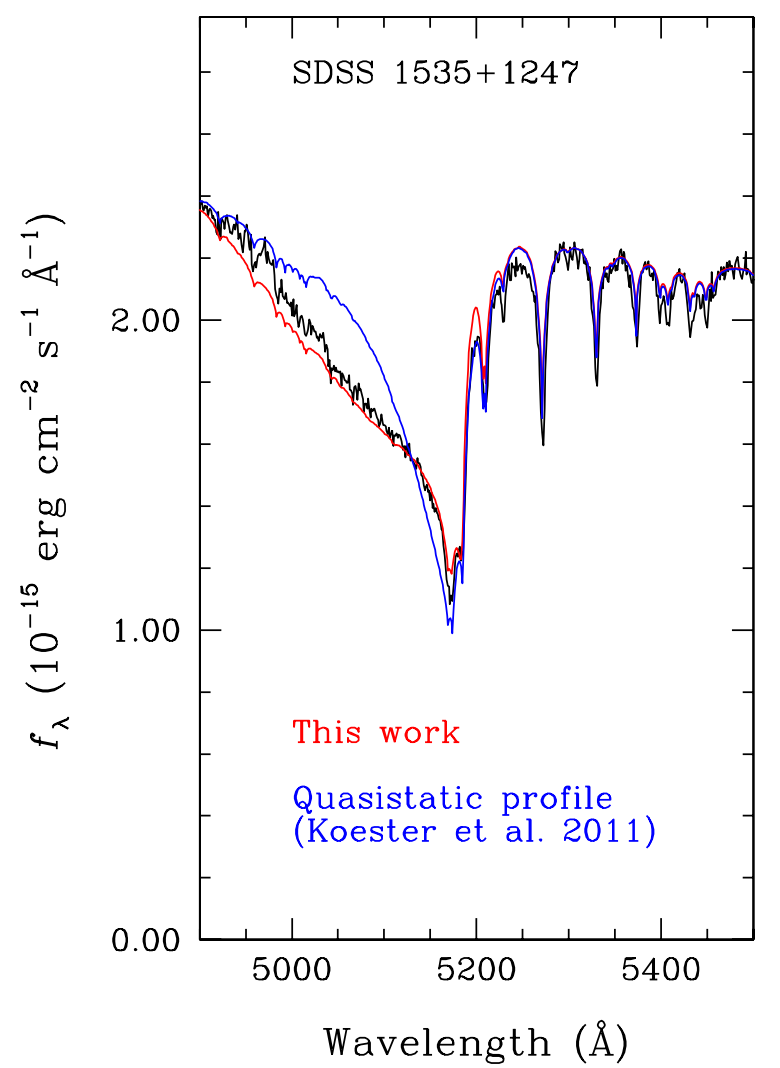

Fig. 13. Comparison of the $\mathrm{Mg}$ line profiles using the quasistatic profile (blue) and the unified line-shape semi-classical theory (red) for SDSS 1535+1247 (black line). The atmospheric parameters of Koester et al. (2011) are assumed.

and shift for $n_{\mathrm{He}}=2 \times 10^{21} \mathrm{~cm}^{-3}$. The asymmetry parameter is then maximum and reaches the value of three.

We see that the profiles and parameters of the triplet $3 p-4 s$ of $\mathrm{Mg}$ perturbed by helium, are thus very sensitive to helium density. They clearly illustrate how non-linear the non-Lorentzian shapes can become outside the limited range of validity of the impact approximation usually used in stellar and planetary atmosphere modeling.

\section{Astrophysical application}

Assuming the atmospheric parameters of Koester et al. (2011) for SDSS $1535+1247$, in Fig. 13 we compare the results from using the unified line shape semi-classical theory to that from the quasistatic line profiles (D. Koester, priv. comm.). We note that the magnesium abundance was increased by a factor of 1.8 relative to Koester et al. (2011) to compensate for the fraction of the core absorption that is now shifted into the blue wing. Further minor adjustments to the atmospheric parameters of Koester et al. (2011), which are beyond the scope of this paper, should further improve the accuracy of the fit to the data. We take the relatively good agreement between the observed line shape of the blue wing and our new calculations as a confirmation that the asymmetry of the triplet $\mathrm{MgHe}$ line is, indeed, due to the blue satellite band that is associated with the $3 \mathrm{p}-4 \mathrm{~s}$ transition.

\section{Conclusion}

We have performed a theoretical calculation of the triplet $\mathrm{MgHe}$ line profiles using a unified theory of spectral line broadening and high quality ab initio potentials. We showed that a 
line satellite band located in the near blue wing of the $\mathrm{Mg}$ lines is responsible of an asymmetry that has not been correctly considered by modelers of cool DZ white dwarfs Wehrse \& Liebert (1980), Koester et al. (2011). This effect of this is increasingly important, with He density, and as a result, the line profiles shifting towards the position of the satellite band. The width, shift, and asymmetry of the lines are very dependent on the nature of the interactions between the atoms at short and intermediate distance when the He density gets high. Whereas the potential energies of Alekseev 2014 ${ }^{1}$ are computed in a smaller range of internuclear distances, the unified profiles are practically the same as those reported here in the extreme physical conditions of cool DZ white dwarfs.

Previously, Allard et al. (2013) discussed the major importance of close blue satellites that are associated with He-He collisions, the line profiles of the $2 \mathrm{p}-3 \mathrm{~s}$ transitions exhibit a similar blue asymmetric behavior. For others alcaline earth metals, similar effects can be expected since comparable electronic distributions and interactions with He could be expected. Therefore asymmetric shapes due to close blue satellites can also be expected for them, in particular for $\mathrm{Ca}$ broadened by collisions with $\mathrm{He}$, the heavier neighboring alcaline earth metal.

Acknowledgements. The authors thank the referee, Paul Barklem, for helpful comments and suggestions that have improved the clarity of the paper. One of us (NFA) would like to acknowledge V. Alekseev for kindly providing the $\mathrm{Mg}-\mathrm{He}$ molecular potentials and dipole moments before publication.

\section{References}

Allard, N. F. 1978, J. Phys. B: At. Mol. Opt. Phys., 11, 1383

Allard, N. F. 2012, J. Phys. Conf. Ser., 397, 012065

Allard, N. F. 2013, in EAS Pub. Ser., 63, 403

Allard, N. F., \& Alekseev, V. A. 2014, Adv. Space Res., 54, 1248

Allard, N. F., \& Biraud, Y. G. 1980, J. Quant. Spectr. Rad. Trans., 23, 253

Allard, N. F., \& Kielkopf, J. F. 1982, Rev. Mod. Phys., 54, 1103

Allard, N. F., Royer, A., Kielkopf, J. F., \& Feautrier, N. 1999, Phys. Rev. A, 60, 1021
Allard, N. F., Deguilhem, B., Gadéa, F. X., Bonifaci, N., \& Denat, A. 2009, Europhys. Lett., 88, 53002

Allard, N. F., Bonifaci, N., \& Denat, A. 2011, EpJ D, 61, 365

Allard, N. F., Monari, A., Deguilhem, B., \& Gadéa, F. X. 2012, J. Phys. Conf. Ser., 397, 012035

Allard, N. F., Deguilhem, B., Monari, A., Gadéa, F. X., \& Kielkopf, J. F. 2013, A\&A, 559, A70

Allard, N. F., Homeier, D., Guillon, G., Viel, A., \& Kielkopf, J. 2014, J. Phys. Conf. Ser., 548, 012006

Anderson, P. W. 1952, Phys. Rev., 86, 809

Cartan, D. G. M., \& Hindmarsh, W. R. 1969, J. Phys. B: At. Mol. Opt. Phys., 2, 1396

Ch'en, S. Y., \& Garrett, R. O. 1966, Phys. Rev., 144, 59

Ch'en, S. Y., Looi, E. C., \& Garrett, R. O. 1967, Phys. Rev., 155, 38

Ch'en, S. Y., Gilbert, D. E., \& Tan, D. K. L. 1969, Phys. Rev., 184, 51

Cohen, J. S., \& Schneider, B. 1974, J. Chem. Phys., 61, 3230

Deguilhem, B., Leininger, T., Gadea, F. X., \& Dickinson, A. S. 2009, J. Phys. B: At. Mol. Opt. Phys., 42, 015102

Fuentealba, P., Preuss, H., Stoll, H., \& Szentplay, L. 1982, Chem. Phys. Lett., 89,418

Fuentealba, P., Stoll, H., Szentpaly, L., Schwerdtfeger, P., \& Preuss, H. 1983, J. Phys. B, 16, L323

Garrett, R. O., \& Ch'en, S. Y. 1966, Phys. Rev., 144, 1

Garrett, R. O., Ch'en, S. Y., \& Looi, E. C. 1967, Phys. Rev., 156, 1

Gilbert, D. E., Allard, N. F., \& Ch'en, S. Y. 1980, J. Quant. Spectr. Rad. Trans., 23, 201

Hrivnak, D., Kalus, R., \& Gadéa, F. X. 2005, Europhys. Lett., 71, 42

Jura, M., \& Young, E. D. 2014, Rev. Earth Planet. Sci., 42, 45

Kawka, A., Vennes, S., \& Thorstensen, J. R. 2004, AJ, 127, 1702

Khemiri, N., Dardouri, R., Oujia, B., \& Gadéa, F. X. 2013, J. Phys. Chem., 117, 8915

Kielkopf, J. F., \& Allard, N. F. 1979, Phys. Rev. Lett., 43, 196

Koester, D., Girven, J., Gänsicke, B. T., \& Dufour, P. 2011, A\&A, 530, A114

Koester, D., Gänsicke, B. T., \& Farihi, J. 2014, A\&A, 566, A34

Leininger, T., Gadéa, F. X., \& Allard, N. F. 2015, in SF2A-2015: Proc. Annual meeting of the French Society of Astronomy and Astrophysics, eds. F. Martins, S. Boissier, V. Buat, L. Cambrésy, \& P. Petit, 397

Paquette, C., Pelletier, C., Fontaine, G., \& Michaud, G. 1986, ApJS, 61, 197

Royer, A. 1980, Phys. Rev. A, 22, 1625

Tan, D. K., \& Ch'en, S. Y. 1970, Phys. Rev., 2, 1

Wehrse, R., \& Liebert, J. 1980, A\&A, 86, 139

Werner, H. J., \& Knowles, P. J. 1985, J. Chem. Phys., 82, 5053

Werner, H. J., \& Knowles, P. J. 1988, J. Chem. Phys., 89, 5803

Werner, H.-J., Knowles, P. J., Knizia, G., et al. 2012, MOLPRO, version 2012.1, a package of ab initio programs 